\title{
El acontecimiento de la escritura La enunciación de Sigmund Freud
}

\author{
The event of writing \\ The enunciation of Sigmund Freud
}

\section{Ángel Fernández}

\section{RESUMEN:}

En este artículo se propone una lectura del ensayo "De guerra y muerte. Temas de actualidad" de Sigmund Freud que pone el foco en la enunciación freudiana, a partir de una serie de preguntas: ¿Qué es lo que hace Freud al producir este escrito? ¿Qué hace con lo que escribe?

A diferencia de las hipótesis de lectura realistas que interpretan el ensayo anterior como una toma de posición del psicoanálisis respecto de la Primera Guerra Mundial, es decir, que lo leen como un análisis de los hechos del momento y por lo tanto, como un "texto de actualidad" ligado al momento de producción; aquí se propone otra vía de acceso que reconoce una operación de anudamiento entre el acontecimiento que inicia el siglo xx y el descubrimiento del inconsciente, en la que ambos (de maneras muy distintas e incluso opuestas) dejan a la luz problemas similares.

Para desplegar dicha hipótesis de lectura, se organizó la escritura del artículo en una serie de fragmentaciones argumentales que mencionaremos a continuación. Ellas son: Atmósfera / Crisis / Sospecha, Superyó /Cuerpo, Estilo / Padre.

\section{SUMMARY:}

This article proposes a reading of Sigmund Freud's 'Thoughts for the Time of War and Death'. Such essay places the focus on the Freudian enunciation from a series of questions: What is it that Freud does when presenting this article? What does he do with what he writes?

Unlike the reading hypotheses from a realist view which propose an interpretation of the mentioned essay as a stance of psychoanalysis towards World War I, that is to say, as an interpretation of the facts from that moment and, therefore as a 'current text' connected to its moment of production, what is proposed here is an alternative approach which acknowledges an operation of knotting between the event that opens the 20TH century and the discovery of the unconscious, in which both (in different and even opposite ways) bring to light similar problems.

In order to put forward this reading hypothesis, the article is organized in a series of argumentative fragmentations, namely: Atmosphere / Crisis / Suspicion, Supergo /Body, Style / Father. 
Palabras clave:

Sigmund Freud - De guerra y muerte. Temas de actualidad - Enunciación freudiana - Inconsciente
WORD PAD:

Sigmund Freud - Thoughts for the Time of War and Death - Freudian enunciation The Unconscious 


\section{INTRODUCCIÓN}

De guerra y muerte. Temas de actualidad es el título que reúne dos ensayos de Freud escritos en I9I5. Seis meses después de que la Primera Guerra Mundial, en palabras de Eric Hobsbawm, marcara el inicio del siglo xx. Volver sobre ellos, ahora que estamos profusamente informados sobre los horrores que le dieron forma a nuestra época, implica no sólo constatar cierta lucidez premonitoria sino también la virtud indicativa de la prosa freudiana. Esta relectura es una ocasión para recordar la vocación de futuro del psicoanálisis y la vigencia de ciertos conceptos que nos resultan fundamentales para entender los callejones sin salida del malestar contemporáneo. Por otra parte, el “(...) ensayo es lugar más apropiado para reunificar razón y estilo: donde el instrumento más idóneo para pensar es, a la vez, el más estilizado" (Alfón, 20I6, p.9). Es justamente en este modo de decir y de pensar en el que nos encontramos con las preguntas que surgen de la impronta ensayística en Sigmund Freud. Particularmente, esa interrogación polémica y virtualmente inextinguible: ¿cómo leerlo?

A diferencia de los textos mayores de la doctrina analítica y el arduo trabajo de interpretación que requieren, los dos ensayos de los que nos ocuparemos parecieran poder interpretarse con facilidad ya que abordan temas sobre los que todo el mundo estaría al tanto. Nos sumergen así en una ilusión de transparencia. Sin embargo, no debemos dejarnos engañar por esa primera impresión. Porque si bien es cierto que hay momentos en los que Freud escribe ceñido a los títulos que propone, existen otros en los que utiliza los temas de actualidad, para seguir desplegando tesis fundamentales de psicoanálisis. En esta línea, plantea tres cuestiones centrales que recorren de una u otra manera toda su obra: la crítica a las ilusiones, la formación de la eticidad y el peso de la moral cultural. La particularidad que presenta en esta ocasión el tratamiento de estos temas, que serán abordados hasta sus últimos escritos, es que la Primera Guerra Mundial aparece como el telón de fondo sobre el que se despliegan. La hipótesis de lectura es que lejos de ser casual, ello responde a un cálculo retórico que pretende articular e incluso sincronizar, el psicoanálisis y la guerra. Como si tanto uno como la otra, de modos completamente diferentes, vinieran a derrumbar los mismos ideales. Como si las tesis del psicoanálisis vinieran a ser confirmadas por los horrores de la guerra y Freud dijera que aunque los círculos intelectuales lo nieguen, lo real está de su lado.

En estrecha relación con lo anterior, es interesante destacar el contraste que plantea la posición de "De guerra y muerte. Temas de actualidad" respecto de la posición de un sabio indignado por la incultura de la humanidad. Dispuesto a hacer propuestas elevadas, a dar sermones y a hacer apelaciones a los buenos sentimientos, o por el contrario, a lamentarse por la maldad intrínseca del hombre. Por el contrario, Freud no parece en ningún momento sorprendido ni rendido argumentativamente, por lo que está ocurriendo; sino que por el contrario, convierte el acontecimiento catastrófico en materia de reflexión psicoanalítica. Pero no es suficiente, va más allá y lejos de mostrarse como un hombre piadoso, le quita a los desilusionados el derecho a estarlo que les facilitaba la vida con engaños. 
En lugar de detenerse en analizar los hechos para ofrecer tentativas de solución, Freud, describe la atmósfera de crisis, sospechando que su límite no se alcanza a percibir con claridad. De este modo, convierte el contexto en texto recordando tanto las pulsiones destructivas que habitan al hombre como la fragilidad y la dependencia del intelecto. En otras palabras, vuelve a ensayar un discurso sobre su teoría de la libido y la articulación con el problema de la percepción y el sentido. Por último pero no por ello menos importante, sin necesidad de explicitarlo, en su argumentación se lee la pregunta por el padre, en un tiempo caracterizado por la caída de los semblantes y la inminencia correlativa de un empuje al goce, en el que el concepto de superyó dinamiza las elucubraciones sobre la pulsión de muerte.

Una lectura que permite ejemplificar de manera concreta y pertinente lo que acabamos de mencionar es la carta que Albert Einstein, con fecha del 30 de julio de I932, le envía como representante de la Liga de las Naciones y del Instituto Internacional de Cooperación Intelectual en París, a Freud a los fines de proponerle un debate cifrado en esta pregunta: “ ¿Hay algún camino para evitar a la humanidad los estragos de la guerra?" (Freud, 20I3). Si bien no nos detendremos en ella porque nos desviaríamos demasiado del tema que nos convoca, no obstante, tampoco podemos dejar de señalar dos aspectos que pueden contribuir con nuestra lectura. En primer lugar, pareciera ser que Einstein no está muy al tanto de lo que Freud había escrito diecisiete años antes sobre el tema que lo ocupa. En segundo lugar, la posición del científico con respecto a la cuestión a tratar es completamente diferente a la del psicoanalista. La condición de representante de un organismo internacional y el voluntarismo fatalista de Einstein contrasta ${ }^{1}$ con la sobria actitud de Freud, en los ensayos de I9 I 5 así como también, en la carta que Freud le responde en setiembre de I932.

El contraste anteriormente mencionado nos permite poner aún más en cuestión la ilusión de transparencia explicitada al comienzo de este trabajo. Incluso, podríamos reconocer un principio de opacidad como hilo orientador de nuestro análisis. Ahora bien, partiendo de esta idea, lo que leeremos a continuación seguirá la lógica del gesto freudiano consistente en tomar la Primera Guerra Mundial no como tema, sino como ocasión. Una ocasión significativa más para abordar preocupaciones teóricas cruciales del psicoanálisis que aparecen en otros textos fundamentales (Tótem y Tabú o El porvenir de una ilusión). Por ello, leeremos De guerra y muerte. Temas de actualidad como la ocasión para hacer puntuaciones temáticas que posibilitan su lectura. En ese sentido, consideré oportuna la fragmentación argumental en ocho aspectos que agruparé en tres series. A continuación, desarrollaré brevemente cada uno de ellos.

En primer lugar, la serie atmósfera-crisis-sospecha tiene por objeto ubicar la importancia de este texto en tanto funciona como una radiografía de nuestra época que muestra una especie de diagnóstico del clima ético y de las tensiones morales que se avecinan. A su vez, intentaremos pensar el lugar de la crítica a las (des)ilusiones que localiza Freud, así como también, definir en qué posición se encuentra la crítica dirigida al mismo psicoanálisis. En segundo lugar, proponemos el par cuerpo-superyó como otra referencia ineludible para nuestra lectura (más allá de que no se los mencione en el texto), debido a 
que operan en el horizonte de la reflexión freudiana en la medida en que el primero es el gran protagonista de la guerra mientras el otro, encierra el núcleo de la pulsión de muerte y amenaza con convertirse en el nuevo amo que augura un estado permanente de compulsión generalizada. En tercer lugar, la serie estilo-padre abre la indagación acerca de algunas peculiaridades de la escritura freudiana con la idea de promover - a contrapelo de los discursos que la ubican como pasada de modauna renovación de los estudios freudianos. Sobre todo, donde la cuestión del padre cobra relevancia teórica a través de procedimientos retórico-conceptuales que reponen, al interior de la literatura analítica, lo que otros discursos desechan. El estilo y el padre se encuentran íntimamente vinculados con la caída de los semblantes y el valor de la falla estructural que vehiculizan.

\section{Primera serie}

\section{ATMÓSFera}

En su libro, Sugestión: potencia y limites de la fascinación política, Andrea Cavaletti realiza un profundo análisis de la nouvelle de Thomas Mann, Mario y el mago en el que plantea que "Si es cierto que el sentido de todo relato se encierra, entonces, en una sola palabra, es posible que no haya un relato que, como Mario y el mago, se halle al mismo tiempo tan concentrado y tan disperso en las letras y en el significado del adverbio atmosphärisch. Es posible que no haya otro relato que (...) sepa ser nada más que un recuerdo, pero un recuerdo muy cercano, que nos rodea como un aire o un clima. Y no hay por lo tanto un relato que sea, como este, algo más que un recuerdo, ni un recuerdo del cual sea más difícil librarse. La 'trágica experiencia' de ese viaje a Italia, en los comienzos del fascismo, resiste aún, suspendida y amenazante, en nosotros y fuera de nosotros". A continuación recuerda que suele interpretarse este texto bajo el signo de la potencia de la alegoría: “(...) en el mago sugestionador cavaliere Cipola se reconoce sin duda a Mussolini, y el ejercicio de la hipnosis colectiva que él practica con los habitantes y los turistas de Torre di Venere es la metáfora perfecta del poder autoritario." Pero insiste en que se trata más bien de lo que él llama "tomar cuerpo de una atmósfera”. (CAVAlETti, 2005, p.8)

La palabra atmósfera que, según Cavaletti, encierra el sentido del texto de Mann, resulta significativa para la lectura que se propone aquí de la escritura de Sigmund Freud y su fina sensibilidad literaria. Es sumamente conocida la admiración que el psicoanalista profesaba por el escritor. Pero también, el gusto por la literatura en sí que lo resguardaba de creer ingenuamente en la realidad.

La referencia al clima, al aire, a la atmósfera de un escrito, reside precisamente en lo que "De guerra y muerte. Temas de actualidad" ofrece como retrato de un momento histórico determinado (el nacimiento del siglo $\mathrm{xx}$ ) bajo una expresión contundente que encontramos al final del primer párrafo: "la maldad de nuestra época” (FreUd, 20I3, p.277). En la frase anterior podemos observar lo que ya notamos - recordemos la carta que mencionamos en la introducción de este trabajo- de la escritura freudiana; lejos de una postura idealista o sabia, abre y delimita el campo de operaciones del psicoanálisis como movimiento político y cultural haciendo precisiones sobre los límites de la posible intervención del psicoanalista. 
Vemos repetirse en "De guerra y muerte $(. .$.$) " el gesto de Freud respecto de su$ Traumdeutung, ligando así, la fecha de publicación al inicio del nuevo siglo, con el escrito que nos convoca, publicado quince años después. En este texto pareciera querer sintonizar nuevamente con los tiempos que acontecen, a través del tratamiento que lleva a cabo de esos "temas de actualidad". No podemos dejar de señalar que la operación freudiana consiste justamente en que el acontecimiento que significa esa guerra debe quedar, para él anudado al acontecimiento que significa el descubrimiento del inconsciente.

En sus escritos, Freud insiste y afirma en ese escribir, una actitud que derrumba las acusaciones que piensan las tesis psicoanalíticas como caducas. Muy por el contrario, de lo que está hablando es precisamente del porvenir. Del tiempo que inaugura la guerra, de los ideales que viene a conmover y de las consecuencias simbólicas que tendrá en el futuro. El verdadero tema del ensayo parece ser la atmósfera del siglo por la desilusión y la reacción contra la desilusión. A su vez consiste también en un agudo cuestionamiento de nuestra actitud hacia la muerte.

No debemos dejar pasar, en esta línea de lectura, una referencia sesgada que Freud realiza con respecto a las sospechas que los individuos empiezan a tener con respecto al Estado y al monopolio de la violencia que instala en la sociedad, puesto que este clima de desconfianza generalizada va impactar también y quizá de manera fundamental, en la política y en el problema del estatuto de la autoridad.

Esta transferencia negativa a escala mundial, este sospechar de algunas de nuestras certezas (que hasta hace muy poco estaban firmemente arraigadas) y las consecuencias que tendrá en la civilización, configura lo que llamamos atmósfera del siglo. Campo de operaciones en el que el psicoanalista deberá calibrar una ética, una técnica y las políticas necesarias para sostener su intervención. Este clima tendrá como protagonista principal a un estado permanente de crisis al que habrá que empezar a descifrar en sus paradojas, conjurar en sus efectos y entender en su presencia obstinada, ya que dejará de ser excepcional para convertirse en la regla forzada del orden de posguerra.

\section{CRISIS}

En pocas palabras y con notable contundencia argumental, Martín Cerda conecta de manera indisoluble al siglo pasado con un persistente sentimiento de crisis: " $\mathrm{La}$ historia del siglo xx resultaría, en rigor, incomprensible y, por consiguiente, parcialmente irrelatable, si excluyéramos de ella ese continuo sentimiento que, desde los mismos inicios de la centuria, ha tenido el hombre de vivir en una constante crisis" (CERDA, 2005, p.I86). Distingue además, entre aquellos cambios que ocurren en el mundo y para decirlo en palabras de Ortega, los cambios del mundo. Estos últimos, de los cuales los hombres suelen percatarse un poco tarde y que afectan las creencias colectivas, nos dejan a la intemperie, desorientados y atrapados en una aporía, vocablo “(...) con que se denominó el problema, la cuestión, la dificultad, es decir, aquello que se presenta a la mente obliterado, que no deja paso a nuestra comprensión” (CERDA, 2005, p.I89). No podría describirse de manera más precisa y sintética, la experiencia del mundo de los habitantes de ese tiempo, de esta atmósfera a la que alude Freud en "De guerra y muerte. Temas de actualidad". 
Una vez más, mientras que la guerra pone en crisis el sistema de creencias que funcionaba hasta el momento en lo irrefutable de su presencia horrorosa; el psicoanálisis por su parte, también lo hace pero bajo la forma — mucho más amable- de la argumentación racional de una experiencia ética inédita que es comandada por la hipótesis del inconsciente, y sostenida por el deseo del analista, que parecía ser indistinguible del propio deseo de Freud en esta época. Y esto en la medida en que abogaba por la apertura de "otra escena" en el discurso, de otro modo de alojar la demanda del sufriente - diferente con respecto a otros dispositivos — sacerdote, médico, pedagogo, por solo mencionar algunos- en los cuales siempre se dispone de antemano de un Bien universal que funciona eficazmente como ideal colectivo. Así, la invención freudiana del psicoanalista pone en suspenso la cuestión del bien, no sin redirigirla a la singularidad y articularla con la brújula que supone el malestar en la cultura. Como si el malestar mismo fuera la vía regia para la pregunta por el bien de cada uno.

Poner en crisis la suma de los prejuicios de una época tiene un alto costo para el psicoanálisis. Su propio mensaje le volverá bajo la forma de un intento sistemático de poner en crisis al psicoanálisis mismo. De este modo encontraremos todo tipo de argumentos revisionistas y anexionistas que buscan socavar la credibilidad de una práctica precisa mientras predican la credulidad hacia otras difusas.

Es justamente en este clima de incertidumbre y de sospecha, en el que el discurso freudiano hace su apuesta de elucidación, sin esperanza y sin pesimismo. Puesto que este clima enrarecido e inestable, extrañamente y contra todos los pronósticos, pue- de ser el campo fecundo tanto para instalar la certeza del inconsciente como para convertir la adversidad de la crítica en un aliado impensado.

\section{Sospecha}

A menudo, nos encontramos con una larga serie de reproches dirigidos al psicoanálisis, provenientes de los más diversos lugares teóricos. Hay uno en particular, que está marcado por el cientificismo posmoderno y referido a la caducidad de los conceptos freudianos para abordar la complejidad de las problemáticas que presenta el mundo actual, que ha cobrado especial relevancia en estos tiempos.

Esta misma línea que critica, también hace referencia a nuevos modos del malestar en la cultura que no estarían contemplados en la reflexión inaugurada por " $\mathrm{La}$ interpretación de los sueños”. Además, se ataca a ciertos modelos del pensamiento freudiano que habrían sido refutados por los avances producidos en el campo de las ciencias humanas y de la ciencia en general. Incluso algunos psicoanalistas insisten, cada tanto, en arrojar dudas sobre Freud y preguntar si, en realidad, no quedó encorsetado en los límites de la estructura de la histeria, no pudiendo captar así otras derivas de la subjetividad contemporánea. Ahora bien, el análisis minucioso que requeriría cada una de estas críticas excede ampliamente los alcances de este escrito. Razón por la cual, solo presentaremos una breve y somera lectura de estos argumentos adversos para así poder ubicar en qué posición se encuentran respecto de la letra freudiana.

Sin embargo, nadie propone dar una vuelta de página respecto al psicoanálisis para pasar a otra cosa. Sólo una pequeña proporción promueve olvidar el psicoaná- 
lisis. Es sumamente difícil encontrar algún discurso que no contenga, aunque sea, una referencia tácita al mismo. ¿Entonces? ¿Cuál es la posición de la crítica? ¿Es posible ubicar al menos una? ¿Y cuál sería su utilidad?

Jacques Lacan propuso una cifra de su relación con Sigmund Freud bajo el significante debate, poniendo el acento en la exposición de las preguntas y las diferencias que apuntaban a la construcción de una red argumental clara y distinta de los supuestos en juego. En oposición al vínculo religioso que los psicoanalistas de la IPA mantenían en relación a la letra freudiana y que los conducía a hacer un respetuoso silencio resguardado en la suficiencia del saber supuesto. "Las Suficiencias no hablan" ironizaba Jacques Lacan refiriéndose a los mismos que decían que su formación era insuficiente para poder dedicarse a la enseñanza en la institución oficial. Hecho que dió argumentos a los "suficientemente" formados, para prohibirla.

Este rodeo por las políticas internas del psicoanálisis nos habilita a volver con un interés renovado a nuestras preguntas, ya que permite situar dos cuestiones articuladas que abren una perspectiva precisa para entender nuestro tema. Por un lado, Lacan le reprocha al Maestro vienés la estructura religiosa de la institución analítica y plantea que hay algo en el deseo de Freud que no está analizado. Por otro, dice encontrarse en un estado de transferencia negativa, es decir que, el texto freudiano se encuentra para él, bajo sospecha. Este es el aspecto que me interesa en este trabajo.

Al revés de la posición de Paul Ricoeur que ubica a Freud como un "maestro de la sospecha”, en la posición lacaniana el sospechoso es el mismo Freud. Entiendo ésta como la posición general de la crítica: la transferencia negativa, es decir, el psicoanálisis bajo sospecha. Este es el punto en el cual, después de un largo rodeo, podemos volver a nuestro texto. Como si se tratara de un caso de defensa anticipada, en el que el escritor preveé las críticas futuras y trata de disiparlas antes de que aparezcan, el énfasis de la cadena de razonamientos que la escritura freudiana pone en marcha, presenta un acuerdo relativo respecto a lo que está en juego en el aire de la época: se trata de la sospecha, sin duda, como derrumbe de las ficciones simbólico-imaginarias que lo real de la guerra viene a plantear.

En discrepancia con la posición de Ricoeur, en la que pareciera que es el psicoanálisis el que sospecha y la posición de Lacan en que es el psicoanálisis el sospechoso, nuestro texto muestra que la sospecha recae ahora sobre la cultura misma. Lo que significa que todo el mundo pone en duda a todo el mundo. Pero hay una vuelta de tuerca más: este clima de confiada desconfianza generalizada no se contradice, y no se contradice en absoluto, con la irrupción de certezas inamovibles. Estas certezas nunca son ajenas al rechazo del cuerpo. Si leemos con paciencia, vamos a ver que todo el tiempo Freud está hablando del cadáver (del enemigo o del ser querido), del intelecto autónomo (del cuerpo) como centro del individuo, del olvido de las raíces afectivas del pensamiento, de las ilusiones que proporcionan un confort precario y ruinoso. En otras palabras, no cesa de hablar del cuerpo y de las múltiples formas de rechazo de la que es objeto. Como si dijéramos que a la célebre fórmula del primer Heidegger de "Ser y Tiempo" alrededor del "olvido del ser”, debiéramos anexarle la también 
temprana fórmula freudiana del "olvido del cuerpo".

\section{Segunda serie}

\section{Cuerpo}

La escritura freudiana siempre supo que sufriría un rechazo ancestral heredado. Así, nació y se desplegó, bajo el signo de la ojeriza de los poderes constituidos, sabiendo que la hostilidad hacia sus teorías no solo no se atenuaría con el paso del tiempo y el avance de su argumentación, sino que incluso se volvería más intensa en el futuro. Sigmund Freud anticipándose a una larga lucha para legitimizar las tesis del psicoanálisis, mediante "De guerra y muerte. Temas de actualidad", hace posible entender algo complejo: no es el psicoanálisis el que tiene mucho que decir sobre la guerra sino exactamente al revés. Es ella, en acto, la que dice.

Por eso, en estrecha relación con lo anterior propongo tomar distancia de las interpretaciones que se inclinan por leer este texto como una toma de partido de Freud hacia la realidad de su época y su obvia preocupación por el estallido de la Gran Guerra. De modo que los conceptos vertidos allí responderían a una coyuntura precisa vinculada al clima general de beligerancia que se vivía en I9I4.

Sin embargo, esta lectura presenta un problema. Deja en las sombras el trabajo textual que el creador del psicoanálisis pone en escena. En efecto, a mi entender, es menos un escrito de época que un manifiesto analítico disfrazado de actualidad. Desde el título mismo hay un juego con la ambigüedad del vocablo "actualidad" como "aquello que pasa en tiempo presente" o "el suceso que llama la atención". Y si seguimos el hilo de la su escritura, también podemos deslizarnos hacia "lo que (del pasado) se actualiza en un presente determinado".

Pero también, en la primera parte del título de este trabajo advertimos una interesantísima operación metonímica. Si bien anuncia de manera clara y contundente que nos va a hablar de dos temas de ineludible actualidad que interesan a todo el mundo (guerra y muerte), apenas comenzamos la lectura - y esto se hace cada vez más notorio conforme avanzamos en ella-, advertimos que en realidad el tema que domina de punta a punta la elocuencia formal del texto es el psicoanálisis.

El "timbrazo de los títulos" que recordaba Oscar Masotta en referencia a "Radiofonía y Televisión” de Jacques Lacan, no debe hacernos olvidar que la argumentación avanza en un territorio desplazado donde, de modo magistral, la "joven ciencia" queda anudada nada menos que a la guerra y a la muerte. Como si estas dos realidades humanas pusieran a cada uno frente a una verdad a la que descubre aquella.

A esta altura, ya no se trata de las resistencias contra el psicoanálisis, sino de lo que viniendo de lo real no nos permite sustraernos a su influjo. Como el dolor de muelas que nos hace olvidar el amor. Lo que este texto trabaja es aquello a lo que en principio no podemos oponerle nada. Es más bien lo contrario: es "eso" lo que opone una tenaz resistencia a la insidiosa necedad de nuestras ilusiones. Todo el hilo argumental se inclina a mostrar la profunda conmoción que la guerra produjo en nuestras certezas y la puesta en evidencia de nuestra actitud ante la muerte. Lo que significa que no se trata de una teoría como entramado simbólico-imaginario 
que intenta ceñir algo de lo real sino que, insistimos, es exactamente lo opuesto. Algo que se presenta como una irrupción inesperada, pero sobre todo inasimilable, que desbarata nuestras teorías sobre nosotros y sobre los otros. Ahora, decepcionados, notamos que bajo nuestros pies, el suelo tiembla.

En ese sentido, es evidente que este texto no está de ningún modo desvinculado de otros que vehiculizan preocupaciones similares. Por lo que forma parte de aquellos que apuntan al señalamiento de un despertar a una realidad (teórica) desde siempre rechazada. "Esta guerra - le escribe a Frederik van Eeden- hace que me atreva a recordarle dos tesis sustentadas por el psicoanálisis que indudablemente han contribuido a su impopularidad.”. En el párrafo siguiente recuerda que “(...) el psicoanálisis ha llegado a la conclusión de que los impulsos primitivos, salvajes y malignos de la humanidad no han desaparecido en ninguno de sus individuos sino que persisten, aunque reprimidos, en el inconsciente (para emplear el término de nuestro lenguaje), y que esperan las ocasiones propicias para desarrollar su actividad."Continúa: "Nos ha enseñado también que nuestro intelecto es una cosa débil y dependiente, juguete e instrumento de nuestras inclinaciones pulsionales y afectos, y que todos nos vemos forzados a actuar inteligente o tontamente según lo que nos ordenan nuestras actitudes (emocionales) y resistencia internas."

Ambas tesis, deben su "impopularidad" al hecho de poner en cuestión dos ideales convergentes. Por un lado, aquel que pretende declarar el triunfo pleno de la cultura sobre el individuo en la implantación de los valores morales que lo vuelven bueno y apto para la vida en sociedad. Por otra parte, se trata del ideal de autonomía del intelecto y de la omnipotencia de la razón. Notemos que tanto uno como el otro dejan fuera de juego un pensamiento sobre el cuerpo, o más exactamente la intervención del cuerpo en el orden del pensamiento.

En este punto es importante recordar el lugar que la filosofía moderna le otorgará a la razón. Reflexión desvinculada del cuerpo que culmina con Descartes y que había iniciado San Agustín, proponiendo una interioridad del sujeto que alojaba al pensamiento como actividad espiritual. Aquí el debate filosófico - y Jacques Lacan es un ejemplo a seguir en ese sentido- es de suma importancia para el psicoanálisis. Sin internarnos en la complejidad del mismo, recordemos simplemente las objeciones de Schopenhauer a esta cuestión con su noción de Voluntad como esencia del mundo. Pero también, y de manera particular, el conocido escolio a la proposición 2 de la Tercera parte de la Ética donde Spinoza plantea lo siguiente: “[...] a nadie ha enseñado la experiencia, hasta ahora, lo que puede un cuerpo [...] Pues nadie hasta ahora ha conocido la fábrica del cuerpo" (SpINOZA, 2005, p. I I3). Esta última expresión con sus múltiples resonancias va a encontrar un contrapunto en el Trieb freudiano. Ese concepto fundamental del psicoanálisis que conecta y separa lo psíquico y lo somático, lo animal y lo humano, el lenguaje y el goce.

Una indagación genealógica que reponga la arquitectura de los debates en el interior de la filosofía sobre este punto, abriría una zona posible de debate fecundo con la historia del movimiento analítico que permitiría esclarecer el lugar que se le otorga al cuerpo en el discurso. Este elemento expulsado de la reflexión teórica es lo que retorna con la guerra, con la muerte (del 
otro) y con el psicoanálisis. También, debemos apuntar — si bien no es el único-a Nietzsche, en su trabajo crítico de la moral ascética y el correlativo desprecio por este mundo.

Recapitulando, se vuelve aún más claro lo que venimos observando: lo que se encuentra bajo sospecha por el rodeo que el psicoanálisis ofrece, es en verdad, esta fábrica del cuerpo a la que lúcidamente alude Spinoza. Por su parte, Sigmund Freud cifra y descifra lo rechazado del discurso por la doble vía de la elaboración teórica más repulsiva para sus contemporáneos bajo el título de pulsión de muerte y el trabajo onírico que conduce al deseo indestructible.

Ahora bien, si hay un concepto psicoanalítico que opera como representante (irrepresentable) simbólico, imaginario y real de las potencias del cuerpo y a la vez de su anulación, es precisamente el de superyó. Su voz inaudible y atronadora siempre susurra lo mismo: ¡Goza! que en el límite es un ¡Muérete! Fragmento de cuerpo que se ha vuelto contra sí mismo. El superyó es la novedad freudiana. No se trata de rechazos que proceden del exterior. No se trata de dispositivos disciplinarios ni de aparatos de control, sino de algo mucho más efectivo. Se trata de la interiorización del rechazo. Se trata de que el cuerpo se rechace a sí mismo. ${ }^{2}$

\section{SUPERYÓ}

El concepto de superyó, a pesar de sus resonancias caricaturescas y el imaginario de cómic que sugiere, ocupa, al igual que el síntoma y la pulsión, un lugar central en el campo analítico. No solo en el ámbito de la reflexión freudiana sino también en la historia del movimiento analítico y su — como diría, otra vez Paul Ricoeurconflicto de interpretaciones. Actúa como un verdadero centro de gravedad que atrae hacia sí las más diversas articulaciones teóricas y las distintas vías de acceso a los conceptos fundamentales del psicoanálisis. El superyó es uno de los nombres secretos del descubrimiento del inconciente en la medida en que es la forma freudiana de decir en una palabra algo que reúne varios problemas a la vez. Entre ellos hay que ubicar la falla de ley, el problema del ( $\sin )$ sentido de los síntomas y la (in) satisfacción que contienen y por último, aquello que constituye el Bien del sujeto en su singularidad. Más precisamente hay que decir que superyó es el nombre que Freud inventa a falta de un concepto que logre ceñir lo que no funciona: de la ley, del sentido, de la satisfacción y del bien en el mundo humano.

¿Pero qué es un mundo humano? En un sentido ideal sería el producto del perfecto funcionamiento de las cuatros cuestiones anteriores. Por supuesto, porque nos educamos en una idea de humanidad que implica el funcionamiento armonioso de la ley regulatoria de la relación entre los seres y a su vez, de cada uno consigo mismo. Una humanidad plena de sentidos plenos y estables compatibles con una satisfacción lograda y bien adaptada al medio social en el que se realiza. En suma, el mundo humano es un mundo en el que hay un Bien que vale para todos y cada uno se orienta por y hacia él.

Es precisamente este ideal de mundo humano el que queda profundamente dañado después de Freud. Y sin, duda es este daño el que provoca las formas más variadas del rechazo hacia el psicoanálisis. Ese mundo humano tan laboriosamente construido a largo de los siglos empezará 
a ser inmundo e inhumano pero a la vez algo completamente nuevo. Y como toda novedad alcanzará lo más antiguo de la civilización para promover una subversión: comenzar a entender lo humano a partir de aquello que no anda o de aquello que anda mal. Esto es: comenzar a interpretar al hombre a partir del síntoma, a los ideales a través de la segregación renegada que producen y a la cultura a partir del malestar en la misma.

Pero, detengámonos un momento para formular una simple pregunta. Este ideal de un mundo humano cuestionado por las tesis analíticas, ¿no es exactamente el mismo que la Primera Guerra mundial viene a destruir? Veamos lo que pasa con un ejemplo tomado de la literatura. Mishima, en un breve pasaje de su novela Música, dice:

El psicoanálisis destruye la cultura tradicional japonesa. La idea oscura y densa de la frustración mancilla la simple y sana vida espiritual de los japoneses. La prudencia de esta cultura siempre ha evitado entrar de forma indiscreta en el corazón humano. Por el contrario, la sucia y vulgar doctrina del psicoanálisis, que afirma liberar de la represión encontrando represiones sexuales en todo comportamiento, que es un pensamiento derivado de la degenerada e ínfima mentalidad occidental. Tú eres el lamepiés de un hebreo, un moscardón que pone sus sucios huevos sobre el ánimo noble y puro de la humanidad. ¡Hijo de puta! (Mishima, I964, p.90)

"Cultura tradicional japonesa", "simple y sana vida espiritual", "prudencia” para rematar con "el ánimo noble de la humanidad": son expresiones que ironizan sobre un origen cierto y puro al que hay que custodiar puesto que está amenazado por un mal al que es preciso destruir a cualquier costo: el superyó, empuje criminal (LAurent, 20I3) que jamás es ajeno a la postulación delirante de un origen puro, cierto y sin falla. "La idea de bandera no cuaja con la de mancha", decía Oscar Masotta.

Ahora bien, aquí es donde se hace imprescindible una breve reflexión sobre el estilo en la medida que nos ayudará a orientarnos en la pregunta clave que sostenemos en este artículo: ¿cómo leer a Freud?

Comencemos por apuntar lo que ya habíamos señalado más arriba respecto a la distancia que la escritura freudiana toma tanto del presente, como de la realidad. A diferencia de la posición del sabio que funda su discurso precisamente en los hechos, ésta distancia propone una temporalidad descalibrada y una perspectiva desenfocada. Para Freud —en I9I5, cuando era prácticamente imposible sustraerse del presente y de la realidad- parece tratarse menos de producir un texto para hablar de "lo que está pasando" que abrir una escena de lectura para que lo real inasimilable suelte lo que viene a decir con su presencia horrorosa.

En ese contexto, llamaremos estilo al ligero desplazamiento que en la escena del texto convierte la ingenuidad de ver y hablar de "objetos de la realidad" en un discurso que realiza los objetos — teóricosnecesarios para objetar la realidad efectiva como ilusión y afirmar la "otra escena" en la que se mueve la lógica pura y dura en la que se sostiene la estructura.

\section{Tercera serie}

\section{Estilo}

Los títulos de Freud son siempre, sin excepción, altamente significativos. Es sufi- 
ciente una lectura minuciosa de ellos para hacerse una idea de la compleja trama de su obra. También sus textos breves, como por ejemplo, "Sobre la psicología del colegial" - alejados de la densidad argumental y teórica de los escritos mayores- como "La interpretación de los sueños" o "Inhibición, síntoma y angustia” presentan una importancia diferencial que es notable, ya sea para un estudio profundo o para una lectura ocasional.

Títulos, textos breves y escritos doctrinales conforman una serie. Desde el comienzo, los títulos no sólo funcionan como un indicador temático sino que, esbozan la tesis a desarrollar que implica siempre una novedad teórica, una refutación del estado de conocimiento en que se encuentra el tema tratado. En cambio, en los textos breves encontramos aproximaciones a la literatura. Anécdotas personales que disparan las reflexiones teóricas y salen del ámbito de lo particular hacia lo universal. O más precisamente, se trata de una escritura que alcanza, en palabras de Sartre, "lo universal-singular".

La clave está en el tono. Si bien Freud habla desde el lugar del analista que desea compartir la novedad de sus descubrimientos y usa la primera persona para reforzar la construcción de la verosimilitud del relato, deja que se oiga al neurótico sorprendido, hastiado, horrorizado por el "decurso demoníaco de su vida psíquica". Es esta huella de la neurosis enmarcada por un análisis, esta huella de una experiencia desbordante para el sujeto, de la que consigue producir un efecto de sonorización de la dificultad y se espera obtener una ganancia de saber, por la vía de la escritura.

Dificultad de la neurosis y dificultad del psicoanálisis que resuenan en lo es- crito. Esa resonancia entre las letras le otorga realidad a la singularidad del descubrimiento freudiano. Es una forma sutil de hacer sonar el estatuto ético del inconsciente. Tal como afirma Jacques Lacan, semejante estatuto no es óntico - no pertenece al registro de los entes intramundanos ni al ontológico, ni tampoco, al onto-teo-lógico. El estatuto del inconsciente es ético y este estatuto ético se deja oír en el tono de la escritura freudiana hasta el punto de formalizar un estilo. Es ese estilo, precisamente, el que invita a la elucidación de las formaciones del inconciente a la vez que echa luz sobre el trabajo del análisis.

Este tono incrustado en la escritura será un legado para el discurso analítico que siempre operará sobre el quiasmo entre el registro opaco del sufrimiento sin razón y el registro pseudo-matemático de las razones del sufrimiento. Este legado -incrustación en la escritura de un tono, que a su vez formaliza un estilo y articula sufrimiento y razón - es lo que convierte a esa manera de decir que tienen los textos freudianos, en una pieza exquisita para cualquier lector. Por ello, jamás falta, en los escritos doctrinales -que presentan las articulaciones conceptuales que habilitan una lectura orgánica del corpus analítico conforme a la experiencia del inconsciente- el compromiso racionalista de los títulos ni la sutileza de los textos breves.

Habría que agregar a esta serie la correspondencia que Sigmund Freud mantuvo a lo largo de su vida con los más diversos interlocutores. Este territorio de lectura ha sido poco explorado en principio, por parecer inabarcable. Cartas que abordan los temas más diversos, desde pequeñas notas de viaje que dan detalles sobre el clima de una pequeña ciudad, noticias sobre el estado de salud de un ser 
querido, pasando por pequeñas confidencias que hablan de la posición teórica de Freud y de lo que él consideraba desviaciones de la misma; hasta llegar a esos comentarios precisos, que afectan y comprometen la ética, la técnica y las políticas del psicoanálisis. La segunda razón por la que no se ha emprendido un estudio sistemático de la correspondencia freudiana reside en que se la ha leído con cierto prejuicio, creyendo que sólo se trata de una serie de anécdotas y cuestiones personales que no revisten valor teórico alguno. La refutación de este prejuicio se encuentra nada menos que en el intercambio epistolar inaugural de Sigmund Freud con Wilhem Fliess en donde encontramos indicaciones clínicas preciosas que ayudan a leer los textos canónicos.

Por las razones antes explicitadas, debemos entender y aceptar que la correspondencia de Freud no es sólo una exquista pieza de literatura analítica sino también y fundamentalmente, un punto de apoyo para entender el psicoanálisis más allá de hipnotizarse con sus dificultades.

Ahora bien, si regresamos a la potencia de los títulos freudianos, podemos observar que la noción de superyó, falta curiosamente en "El yo y el ello". Sin embargo, la ausencia de la palabra de lo que, en este caso, constituye la materia misma del escrito, pareciera ser un gesto típico del psicoanalista. Tal vez el punto sea comenzar a preguntarse el por qué de esa falta. Si seguimos la hipótesis del compromiso racionalista de Freud, su inclinación por la exactitud y la precisión en el uso deliberado de la lengua, podemos fácilmente aceptar una astucia, una "virtud indicati$v a$ ”, en la que la ausencia de un vocablo dice lo que no diría su presencia. En efecto, el superyó -y ésta es una definición provisional mínima- es precisamente, lo que no aparece como tal. O mejor dicho, lo que parece no estar ahí. Es una presencia éxtima y susurrante. No es casualidad que Jacques Lacan busque en la psicosis los puntos de anclaje de su interpretación del superyó freudiano particularmente en la voz áfona. Esa voz que mientras menos suena más difícil es sustraerse a ella.

Hay otro texto de los que podemos llamar breves que opera de modo inverso al que solemos estar acostumbrados. Me refiero a "Un trastorno de la memoria en la Acrópolis”. Allí nos encontramos con que en lugar de proponer una tesis para, a continuación, proceder al despliegue de las cadenas argumentativas pertinentes, lo que hace es alojar una historia personal en el interior de una carta dirigida a un amigo. De ella extrae una conclusión universal que expone brevísimamente sobre el final del texto como quien comenta al pasar una sospecha, una intuición, una ligera y amable advertencia.

Contra esa forma de censura consistente en dar algo por ya sabido para desentendernos de la profunda perturbación que nos provoca su irrupción, la táctica freudiana teoriza, no sobre lo cotidiano sino sobre el borde en el que lo cotidiano se interrumpe - no la memoria sino su trastorno- para avanzar hacia la elucidación de los atolladeros del deseo humano. Por eso, recién sobre el final de la carta, Freud formula su tesis: lo que impide ir más allá del padre es un sentimiento de piedad. Retoma así un señalamiento de su amigo y discípulo Sándor Ferenczi quien indicaba que en la clínica psicoanalítica todo ocurría como si se tratara de ir más allá del padre y de la dificultad que presentaba, para cada uno, dicha empresa. 


\section{PADRE}

¿De qué hablamos cuando hablamos del padre y fundamentalmente qué es lo que hacemos al hablar de él? En primer lugar debemos recordar la ecuación —-subrayada por Lacan - función paterna/superyó y la economía en juego en ella. Mientras más presente se encuentre una, más ausente se hallará el otro. Este esquema de $+\mathrm{y}-$ nos permite situar un resorte clave de nuestro texto en cuestión: si bien lo que aparece en primer plano es la caída de ciertas referencias simbólicas que producen una atmosfera generalizada de desilusión, ésto no implica que accederemos a un levantamiento de antiguas creencias que redundará en una mayor libertad para el individuo y para la sociedad. Al contrario, - otra vez emerge la tesis impopular que recordaba Freud- este clima de falta de referencias simbólicas, esta época líquida diríamos hoy con Bauman, puede encaminarse hacia un reforzamiento de la ferocidad superyoica. Del mismo modo que todo lo sólido al desvanecerse en el aire deja paso al frío cálculo utilitario, la conmoción de las ilusiones que Freud anota en De guerra y muerte. Temas de actualidad puede conducir al imperio de la compulsión y el sacrificio a los Dioses Oscuros.

Esta ecuación y su economía son inherentes a la teoría y la práctica del psicoanálisis. Es imperioso decir que nadie detenta ningún saber acumulado sobre el padre en su lugar de falla estructural y menos aún los analistas, que son precisamente los que han debido dejarse enseñar del discurso analítico esta verdad: es en la relación de un sujeto con las fallas del saber, en la disfunción de la palabra, en el fracaso de la intención donde cobra importan- cia cualquier reflexión sobre el padre en la teoría. De modo que el espesor de la argumentación no equivale a poseer ninguna sabiduría sino más bien, a esa constante preocupación y cuidado de Freud por el múltiple interés que tiene el psicoanálisis para otras disciplinas teóricas, pero principalmente para los "temas de actualidad" que le dan forma a nuestra época.

Entonces, hablar del padre es hablar del lugar del discurso en todo lo que hace a la cuestión del sujeto. Parafraseando a Lacan podemos decir que el padre, como la verdad, tiene estructura de ficción. Pero, por otro lado, también es indicar al discurso como el lugar donde ocurren las operaciones que hacen del padre una metáfora del acceso de un hijo a su historia sexuada. Por ello, el lugar del discurso y el discurso como lugar son sin duda las dos indicaciones preliminares a todo tratamiento posible de la cuestión del padre. Por esta razón, debemos apartarnos de cualquier perspectiva sociológica y/o sustancialista que se oriente hacia un realismo del padre, que lo imagine como un objeto productor de predicados, como una entidad positiva de la que pueden inferirse una serie encadenada de atribuciones.

Es justamente a la inversa, debemos investigar cómo y por qué el psicoanálisis localiza en la función/defunción del padre la posibilidad/imposibilidad de que haya alguien, alguna realidad, cierto orden de objetos o cierto "morar en el mundo", en sentido sartreano, de lo que la filosofía llama del orden del ente. Dicho con premura, no se puede hablar del padre sin recordar que lo que decimos y el hecho mismo de decir, está profundamente marcado por su referencia. El padre, aparece de este modo, menos como objeto de estudio que como condición misma de la palabra. 
A la vez debemos reconocer que funciona como un nombre para la investigación sexual infantil. Toda búsqueda es la marca de un corte que inaugura el valor de una falta y toda investigación está tomada en "el enigma del padre". Por esta razón, insistimos en que, después de Freud, es aquello que nos encamina por los desfiladeros de un derrumbe. No es un objeto de investigación sino el efecto de abismo que se produce cuando el objeto se desvanece, cae a pique, se hunde en esa decepción fundamental que es condición de la emergencia del deseo. De allí que en el orden de la argumentación esté, en consecuencia, determinado por las figuras surgidas de la investigación de una ausencia. Es decir, de una ausencia espectacular, que el ojo registra y no registra a la vez. Decimos ausencia pero también, corte, separación, distancia, abismo, derrumbe. ¿De qué modo ordenar este inventario?

¿Y su relación con el malestar en la cultura? Aún nos falta situar esta cuestión. Ello acarrea más y nuevos problemas en torno al modo de ordenar la dispersión de las referencias y a la aparición de relaciones inesperadas. Para decirlo sin vueltas: hablar del padre - promoverlo como objeto de investigación, situarlo como problema, promocionarlo como significante y hasta agitarlo como provocación- es no hablar de la biología como etiología del malestar en la cultura.

De ningún modo hay que pensar esta oposición en términos de rivalidad especular. El psicoanálisis justamente se constituye como el señalamiento de la heterogeneidad radical de ambos órdenes. Para decirlo en términos del Sartre de Lo imaginario, pertenecen a dos órdenes distintos de existencia.
A los fines de apaciguar algún arrebato biologicista o la simple inercia del sentido común es preciso recordar que Sigmund Freud siempre tuvo en su horizonte el ideal de las ciencias naturales como límite a las "visiones del mundo". Pero la explicación físico-química del trastorno mental se desencuentra con la tesis freudiana de la enfermedad como trabajo de restitución nada menos que de la función del padre.

Por otra parte, el argumento progresista-conservador de la declinación del padre y de su necesaria eventual restitución elude plantear el problema difícil y nada tranquilizador del imperio de la madre. Lo que los discursos nostálgicos e indignados ocultan es la pregunta por el lugar actual del poder y sus consecuencias. En otros términos, de tanto hablar de la decadencia del padre se olvidan de preguntarse por aquello que se encuentra en su apogeo, aquello que funciona perfectamente bien, incluso cada vez mejor. Globalización es solo una palabra para empezar a balbucear los contornos de esa potencia para nada ajena a la concentración de capital, que tiene como uno de sus rasgos sobresalientes lo que podemos llamar una notable infantilización generalizada.

Sin embargo, sería simplista y hasta liviano, considerar que los vínculos — para decirlo rápidamente- entre capitalismo y neurosis sean obvios. El frenesí editorial por conectar a Marx con Lacan tampoco ayuda a esclarecer y ahondar en dichos vínculos. Muy al contrario contribuye a oscurecer casi todo, incluso aquellas cosas que habían comenzado a ser despejadas por los textos inaugurales.

Por último, insistamos en que el padre no es estrictamente hablando un concepto, sino más bien el horizonte del concepto. 
Por lo tanto, entendemos que los temas que se proponen a nuestra reflexión pueden adoptar el aspecto de algo ajeno a nuestra investigación, sin embargo, nos resultan ineludibles para la elucidación de nuestro tema central en tanto el mismo está hecho de esas ajenidades. Me refiero, desde luego, al papel del deseo de la madre y de la sexualidad femenina, del Falo y la diferencia de los sexos - del sexo como diferencia-, del complejo de castración y el Edipo, de la articulación entre inhibición, síntoma y angustia, de la Pulsión y la elección de neurosis, del Deseo y la Ley.

Por fin, hablar del padre es estar por entero situado en el campo de investigación abierto por la reflexión freudiana y como está bien dicho, si no hay un texto único dedicado a la paternidad es porque esta cuestión hilvana todos los textos que conforman el corpus teórico del psicoanálisis y hasta nos invita a pensar que el mismo psicoanálisis no es sino una investigación sobre el padre.

\section{A MODO DE CONCLUSIÓN}

La lectura de los ensayos "De guerra y muerte. Temas de actualidad" nos condujo hacia la lógica del fragmento y la sugerencia. Hemos optado por un salto hacia las resonancias del texto en lugar de orientarnos hacia una disciplina del comentario. La elección pretende recuperar algo de la enunciación freudiana. En ella subrayamos una posición alejada de la sabiduría de los notables. Muy por el contrario, en lugar de entregarse a la elegancia ingenua al estilo de “¿Por qué la guerra?” (EInsTEIN, I932), Freud se asume, en un una lectura de su época lúcida y comprometida, como el portador de las malas noticias.
Por otro lado pero en estrecha relación con lo recientemente expuesto, hemos destacado que la astucia freudiana consiste no tanto en tomar a la guerra como objeto de reflexión sino más bien en hacerle decir su verdad. En este sentido los títulos de ambos ensayos son elocuentes: "La desilusión provocada por la guerra" y "Nuestra actitud hacia la muerte". Lo dicho hasta aquí, nos respalda cuando decimos que nos oponemos a la interpretación usual que hace de Freud un escritor de su época y subrayamos la atemporalidad de su escritura. ¿De qué actualidad habla? ¿Acaso es obvio? ¿Es que hubo algún tiempo en el que los temas mentados no hayan sido de actualidad? ¿Cuál ha sido ese tiempo en el que la humanidad no guerreaba ni moría?

Entonces el verdadero tema de actualidad es el psicoanálisis mismo. Paradójicamente, mientras es resistido por los círculos intelectuales, es confirmado por la realidad de la guerra. Como si algo que viene de lo real defendiera las tesis analíticas que se orientaban, desde hacía más de quince años, a cuestionar con argumentos teóricos, la noción de progreso indefinido de la humanidad.

La Primera Guerra Mundial muestra que el yo que no es amo en su propia casa. Ocasión que Sigmund Freud aprovecha con agudeza analítica, para convertir el contexto en texto. De allí que sea digno de notar el coraje de la enunciación freudiana al poner en serie el acontecimiento inaugural de la guerra de I9I4, con ese otro acontecimiento de su autoría que es el descubrimiento del inconsciente. Ambos abren un nuevo horizonte de interrogantes que aún hoy estamos lejos de clausurar.

Por otra parte, su lectura también es notable. En ningún momento se promueve 
como un sabio salvador de la humanidad sino por el contrario, en la agudización del tono ensayístico que mantiene a lo largo del escrito, insiste en el análisis racional de los problemas teóricos de los que ya se venía ocupando, pero esta vez, bajo la luz enrarecida de la guerra

Es por eso que podemos considerar este texto como un esfuerzo de estilo que recrea la atmósfera moral de crisis permanente y de sospecha generalizada en la que se moverá tanto el mundo como la acción analítica a partir de ese momento. En ese contexto, los temas psicoanalíticos y la reflexión que conllevan pasarán a ocupar un lugar decisivo en las encrucijadas del porvenir en la medida en que, tanto la cuestión del padre, las paradojas del superyó - por tomar sólo algunos aspectos de este artículo- pero también el resto de los problemas fundamentales del psicoanálisis, van a reponer el lugar del cuerpo en la teoría. Esta reposición está plagada de consecuencias. Recordemos para terminar, que hay una pregunta que recorre De guerra y muerte. Temas de actualidad: "¿Cómo es imaginado, en verdad, el proceso por el cual un individuo humano alcanza un nivel superior de eticidad?" (FreUd, I 893, p.82) Esta pregunta de genealogista acentúa el modo en el que la imaginación humana produce los ideales. En esa producción queda excluído el cuerpo. Cuestión que la guerra repone de manera horrorosa y el psicoanálisis al revés, bajo el orden del concepto. Esta reposición teórica del lugar del cuerpo en la cultura es un acontecimiento correlativo al descubrimiento del inconsciente. En esta operación Sigmund Freud deja deslizar una afirmación hacia el porvenir: a partir de ahí habrá que pensar casi todo de nuevo.

\section{NOTAS AMPLIATORIAS}

1. Parte de estas diferencias está recogida por James Strachey en su nota introductoria al texto, en la que recuerda, en las palabras que dirige a Eitingon en una carta, la falta de entusiasmo que le producía a Freud operar como interlocutor: “(...) había terminado esa correspondencia tediosa y estéril a la que se dio en llamar discusión con Einstein" (JONES, I957, p. I87).

2. Para una mayor comprensión de este problema véase El desafío del nibilismo. La reflexión metafísica como piedad del pensar de Remedios Ávila, Trotta 2005, pp. I03-I29. 